\title{
ARTÍCULOS
}

\section{Residuos leñosos de gran tamaño en un torrente de la Cordillera de Los Andes, Chile: su funcionalidad e importancia}

\author{
Study on large woody debris in a stream from \\ the Andes Mountains: functionality and importance
}

\author{
Andrea Andreolia*, Giovanni Carlig ${ }^{b}$, Francesco Comiti ${ }^{b}$, Andrés Irouméc \\ *Autor de correspondencia: a Università di Padova, Dept. Territorio e Sistemi Agroforestali, Agripolis, \\ Viale dell’Università No 16, 35020 Legnaro, Italia, Tel.: 39-049-8272700, Fax: 39-049-8272686, andrea.andreoli@unipd.it \\ ${ }^{\mathrm{b}}$ Università di Padova, Dept. Territorio e Sistemi Agroforestali, Agripolis, Italia \\ ${ }^{\mathrm{c}}$ Universidad Austral de Chile, Instituto de Manejo Forestal, Casilla 567, Valdivia, Chile
}

\begin{abstract}
SUMMARY
The study analyzed amounts, characteristics and morphological impact of Large Woody Debris (LWD) in Tres Arroyos torrent of the Chilean Southern Andes draining an old-growth forested basin. All woody debris pieces greater than $10 \mathrm{~cm}$ in diameter and $1 \mathrm{~m}$ in length were surveyed along a $1.5 \mathrm{~km}$-long stream section presenting a general step-pool/cascade morphology. The total amount of large woody debris within the fluvial corridor was on average $1,500 \mathrm{~m}^{3} /$ ha, very high value comparable only to data from old-growth forested basins in the Pacific Northwest of North America. Around two thirds of LWD volume were found in accumulations while half of the LWD elements were located on the active floodplain. As much as $83 \%$ of the LWD pieces showed signs of in-stream transport, $13 \%$ were directly associated to natural tree falls, and the remaining to landslides and bank erosion. Different types of log-jams were observed, some heavily altering channel morphology (log-steps and valley jams), others just lining the channel edges (bankfull bench jams). The percentage of log-steps over the total number of steps is around $22 \%$, whereas the elevation loss due to LWD (log-steps and valley jams) was $27 \%$ the total potential energy. Finally, $2,000 \mathrm{~m}^{3}$ of sediments were estimated to be stored in the main channel behind LWD structures, corresponding to approximately $150 \%$ of the annual basin sediment yield.
\end{abstract}

Key words: large woody debris (LWD), old-growth forested basin, channel morphology, valley jams, stored sediments.

\section{RESUMEN}

Se registraron la cantidad, características e impacto morfológico de los residuos leñosos de gran tamaño (large woody debris o LWD) en el torrente Tres Arroyos, situado en la Cordillera de Los Andes, Chile, cuya cuenca alta está cubierta por bosques primarios de los géneros Araucaria y Nothofagus. Se examinaron los residuos leñosos de más de 10 centímetros de diámetro y $1 \mathrm{~m}$ de longitud, en un tramo de $1,5 \mathrm{~km}$ del cauce principal del torrente que presenta una morfología general de step-pool/cascade. La cantidad de LWD en el cauce y sus márgenes inundables fue de $1.500 \mathrm{~m}^{3} / \mathrm{ha}$, valor comparable a los registrados en cuencas con bosques antiguos en la costa noroeste de América del Norte. Casi dos tercios del volumen de LWD formaban acumulaciones de troncos y la mitad de ellos se encontraron en las márgenes inundables activas del torrente. El 83\% del LWD presentaba señales de haber sido movilizado por el torrente, el 13\% se asociaba a caídas naturales de árboles y el resto a aportes por deslizamientos y erosión de laderas. Se observaron diversos tipos de acumulaciones de troncos que alteraban la morfología del canal y cubrían las márgenes del cauce. Se estimó que las acumulaciones de LWD en el tramo estudiado disipan el $27 \%$ de la energía potencial total de la corriente de agua. Finalmente, se estimó en $2.000 \mathrm{~m}^{3}$ el volumen de sedimentos depositados en el cauce principal aguas arriba de cúmulos de LWD, correspondiendo aproximadamente al 150\% del aporte anual total de sedimentos sólidos de la cuenca.

Palabras clave: large woody debris (LWD), residuos leñosos de gran tamaño, morfología fluvial, acumulaciones, transporte de sedimentos.

\section{INTRODUCCIÓN}

Los troncos y trozos residuales de madera de gran tamaño (diámetro medio superior a $10 \mathrm{~cm}$ y longitud mayor a $1 \mathrm{~m}$ ) retenidos en los cauces fluviales se denominan en la literatura anglosajona como large woody debris o LWD (Shields y Gippel 1995, Darby y Thorne 1995, Montgomery y Buffington 1997, Dudley et al. 1998, Montgomery et al. 2003).

En siglos pasados los LWD fueron abundantes en las cuencas forestadas; sin embargo, durante las últimas décadas su presencia se ha reducido en forma notable debido a la 
deforestación que ha afectado particularmente las laderas de menor altitud de las cuencas de montaña. También ha contribuido a la desaparición de los LWD la extracción de madera desde los cauces (channel gardening), que se realiza para reducir el riesgo hidráulico, mejorar la navegación y utilizar los residuos vegetales como combustible (Montgomery et al. 2003).

Según Gurnell et al. (2002), Montgomery et al. (2003) y Montgomery y Piégay (2003), los trozos de madera que yacen en los canales ejercen una fuerte influencia en la hidráulica de los ríos, en el transporte de sedimentos, la morfología del canal y la ecología del cauce. En opinión de Montgomery y Buffington (1997), la morfología del canal y la acumulación de sedimentos en los torrentes de montaña que atraviesan bosques maduros son determinados en gran medida por los LWD, que establecen una morfología forzada por escalones de troncos (log-steps), diques y acumulaciones de troncos en el canal y sus márgenes (log-jams y bankfull bench jams), que producen pequeñas cascadas escalonadas entre las que se intercalan pozas (steppool/(cascade). Aunque la presencia de LWD representa una seria preocupación por el transporte del flujo en crecidas y flujos aluvionales (Darby y Thorne 1995, Shields y Gippel 1995, Dudley et al. 1998), las ventajas ambientales de los residuos leñosos son tales que en la actualidad se incorporan restos de madera a los cauces para mejorar la calidad de los ecosistemas fluviales (Hilderbrand et al. 1997, Lacey y Millar 2004, Shields et al. 2004).

La mayoría de las investigaciones geomorfológicas sobre los LWD se ha realizado en la región noroeste de Norteamérica, algo en Europa (Comiti et al. 2006) y muy poco en otros continentes (Jacobson et al. 1999, para África; Baillie y Davies 2002 y Webb y Erskine 2003, para Oceanía). Hasta donde el conocimiento de los autores alcanza, no se han reportado estudios sobre el LWD en las regiones templadas y tropicales húmedas del continente latinoamericano, que se caracterizan por millares de cursos de agua que atraviesan bosques nativos y manejados que presentan árboles de grandes dimensiones y donde, por lo tanto, debe esperarse que el LWD ejerza una acción morfológica importante. En esta área tan importante, sólo Montgomery et al. (2003) en una expedición en el río Beni, en la cuenca amazónica boliviana, destacan la presencia de trozos de madera y grandes acumulaciones de troncos. En este contexto, los Andes meridionales parecen una localización ideal para estudiar el efecto de los LWD en los cauces de montaña que drenan cuencas cubiertas por bosques.

Los bosques nativos primarios son cada vez menos abundantes en los Andes meridionales y en las áreas de precordillera tanto de Chile como de Argentina, debido a la tala de árboles para producir madera y leña y para dejar espacio a la producción agrícola y ganadera (Hechenleitner et al. 2006, Otero 2006). Más específicamente, el bosque lluvioso templado chileno, o bosque valdiviano, similar a los bosques del noroeste de Estados Unidos a lo largo de la costa del Océano Pacífico, ha sido eliminado en gran parte en los valles de Chile central y meridional (Donoso 1993). Sin embargo, en los ambientes andinos es aún posible encontrar grandes extensiones de bosques nativos primarios dominados por Nothofagus spp. y araucaria (Araucaria araucana (Mol.) K. Koch.) en parques y reservas nacionales y en propiedad de particulares (CONAFCONAMA 1999), de tal manera que estas localizaciones representan excelentes áreas de investigación para analizar la influencia de los LWD sobre la morfología y la dinámica de los ríos de montaña en ambientes casi prístinos, diferentes a las cuencas norteamericanas. El estudio de los LWD en el torrente Tres Arroyos resulta entonces de gran interés, debido a la escasa información sobre el rol de los residuos leñosos en los torrentes de América Latina y especialmente del sur de Chile. La estrecha relación entre el drenaje torrencial propio de áreas montañosas de alta pendiente y las propiedades erosionables de gran parte de los sedimentos andinos de origen volcánico (cenizas, tefra) acumulados en las cuencas, hace presumible que el rol de los LWD en la configuración de los canales, en la regulación del arrastre de sedimentos inertes y orgánicos y en la configuración del hábitat para los organismos bentónicos, en la zona meridional de la Cordillera de los Andes, sea muy importante.

Este trabajo tiene como objetivo reportar los resultados de un estudio tendiente a determinar la cantidad y las características de LWD en un torrente de la Cordillera de Los Andes (Chile), describir el impacto de LWD sobre la morfología del canal y la producción de sedimentos a nivel de la cuenca, y comparar los resultados con cuencas situadas en otras regiones del mundo.

\section{MÉTODOS}

Área de estudio. El área de estudio corresponde a la cuenca del torrente Tres Arroyos, situada en la Reserva Forestal Malalcahuello-Nalcas, en la provincia de Malleco (figura 1). El torrente Tres Arroyos cruza la Ruta R-89 entre las localidades de Curacautín y Lonquimay, a unos $2 \mathrm{~km}$ al este de Malalcahuello, para luego confluir sobre la ribera norte del río Cautín. El área de la cuenca hasta el punto de descarga en el ápice o extremo aguas arriba del cono de deyección es de 9,1 km². Desde el año 1997 una estación fluviométrica está en operación en la parte alta de la cuenca controlando una superficie de $5,9 \mathrm{~km}^{2}$ (Iroumé 1997, 2003). Aunque se presentan nevadas en el invierno, el régimen hidrológico del torrente Tres Arroyos es dominado por las lluvias invernales con ocasionales máximos por derretimiento de la nieve que se acumula en las partes altas de la cuenca. Los principales parámetros geomorfológicos de la cuenca se presentan en el cuadro 1 .

Según la clasificación de Fuenzalida (1965) el área presenta un clima "Templado-cálido con lluvias invernales", con una precipitación media anual de $2.217 \mathrm{~mm}$. El mes más lluvioso es junio con $402 \mathrm{~mm}$, mientras que el más seco es enero con $57 \mathrm{~mm}$. La temperatura media anual es 
$8,5^{\circ} \mathrm{C}$, siendo enero el mes más cálido con una temperatura media de $14,3{ }^{\circ} \mathrm{C}$ y julio el mes más frío con $3,3{ }^{\circ} \mathrm{C}$.

La geología del área está caracterizada por rocas piroclásticas, como brechas andesíticas, tufos e ignimbritas, lavas, y capas sedimentarias pertenecientes a la Formación Cura Mallín del Mioceno medio-inferior (Emparan et al. 1992). Dos unidades volcánicas más recientes, del Pleistoceno y Holoceno, están asociadas con el volcán Lonquimay. Los sedimentos cuaternarios se describen como "sedimentos no consolidados, con intercalaciones piroclásticas". En el área de Malalcahuello se encuentra solamente un tipo de roca intrusiva, que corresponde sobre todo a monzonitas y granodioritas (Grupo Plutónico Melipeuco).

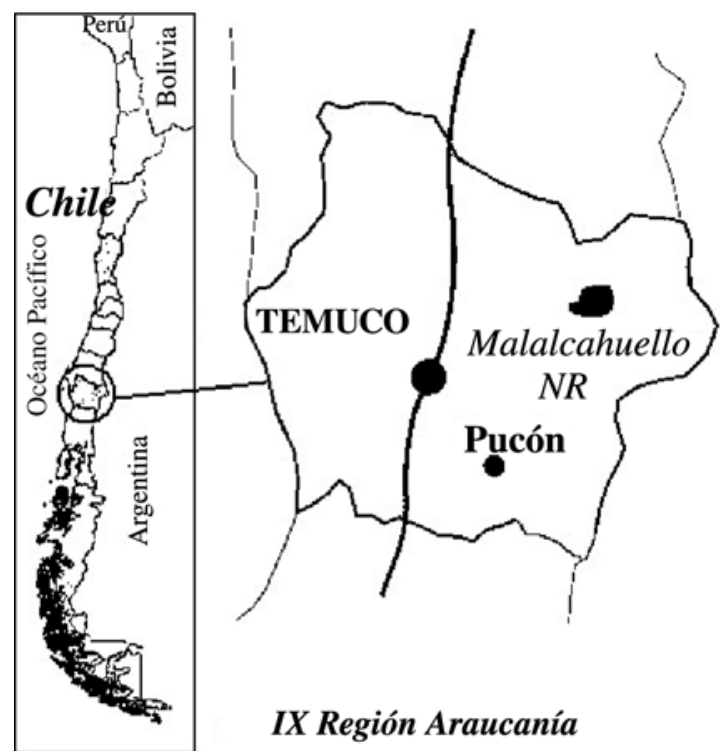

Figura 1. Localización geográfica de la cuenca del torrente Tres Arroyos.

Geographic location of Tres Arroyos study basin.

Cuadro 1. Parámetros geomorfológicos de la cuenca del torrente Tres Arroyos.

Main characteristics of Tres Arroyos catchment.

\begin{tabular}{lcc}
\hline & Unidad & Valores \\
\hline Área & $\mathrm{km}^{2}$ & 9,1 \\
Altura media & $\mathrm{m}$ s.n.m. & 1.487 \\
Altura mínima & $\mathrm{m}$ s.n.m. & 990 \\
Altura máxima & $\mathrm{m}$ s.n.m. & 1.856 \\
Longitud del cauce principal & $\mathrm{km}$ & 4,89 \\
Pendiente media del cauce principal & $\%$ & 18 \\
Orden del cauce & - & 3 \\
Precipitación anual & $\mathrm{mm}$ & 2.217 \\
Caudal máximo registrado* & $\mathrm{m}^{3} / \mathrm{s}$ & 14 \\
Tiempo de concentración & $\mathrm{h}$ & 1,3 \\
\hline
\end{tabular}

* El valor se refiere al registro en la estación que controla una superficie de $5,9 \mathrm{~km}^{2}$ en la parte superior de la cuenca, según se muestra en la figura 3 .
La cuenca se encuentra forestada en un $72 \%$ (figura 2), donde el $61 \%$ es bosque nativo adulto (árboles de 40-50 $\mathrm{m}$ de alto y 1-2 m de diámetro) y el $6 \%$ coníferas. Estas últimas se plantaron para reducir la erosión del suelo después de un incendio ocurrido durante la primera mitad del siglo pasado. El 6\% de la cuenca se caracteriza por cenizas volcánicas arenosas en las partes altas sobre el límite altitudinal de la vegetación y el $22 \%$ se encuentra cubierto por praderas y matorrales cerca del límite de los árboles. Los bosques nativos en la cuenca del estero Tres Arroyos corresponden a los tipos forestales Araucaria (Araucaria araucana) y Roble-Raulí-Coigüe (Nothofagus dombeyi (Mirb.) Oerst., Nothofagus alpina (Poepp. et Endl.) Oerst. y Nothofagus obliqua (Mirb.) Oerst., respectivamente. En el área de la cuenca, el tipo araucaria se encuentra solamente por encima de una elevación de 1.200-1.300 m, mientras que la parte más baja está caracterizada por el tipo Roble-Raulí-Coigüe (DGA 2000). El sotobosque de esta última formación está dominado por quila y coligüe (Chusquea spp.). En las plantaciones destacan las de pino oregón (Pseudotsuga menziesii (Mirb.) Franco) y pino insigne (Pinus radiata D. Don), encontrándose también rodales con $P$. ponderosa Dougl., $P$. monticola Douglas ex D. Don, P. contorta Dougl. ex Loud y una variedad de Eucalyptus spp.

El canal principal es de aproximadamente cinco kilómetros de largo, con una pendiente media del 18\% (figura 3). Sin embargo, el gradiente varía localmente debido a deslizamientos, grandes acumulaciones de residuos en el valle (large valley jams, debris dams) y en el cauce (log-steps) que dan a la sección longitudinal un perfil escalonado. La morfología del cauce (según la

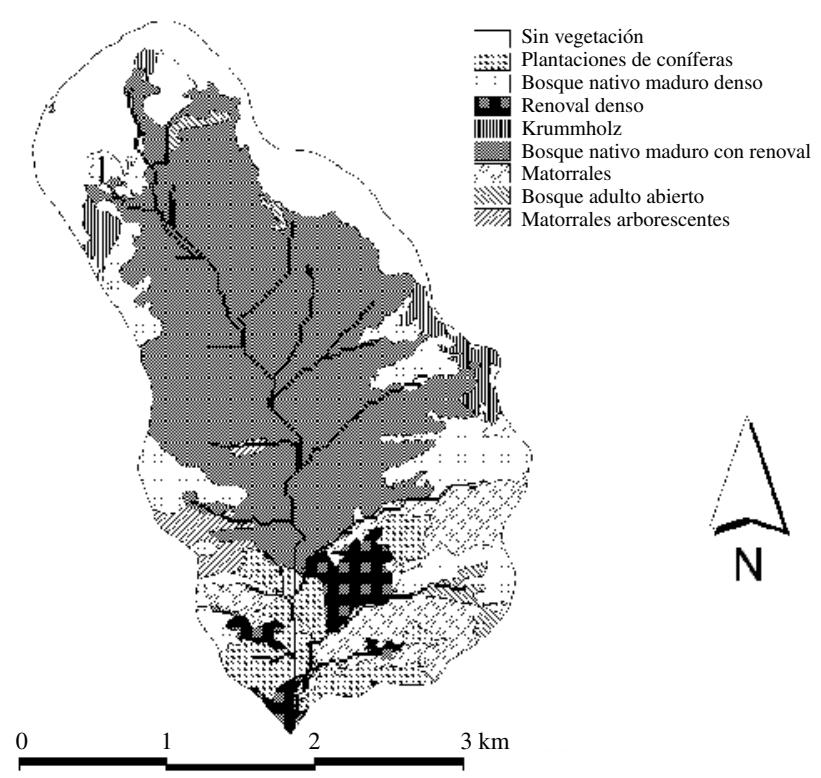

Figura 2. Mapa de cobertura vegetal de la cuenca del torrente Tres Arroyos.

Vegetation map of Tres Arroyos basin. 


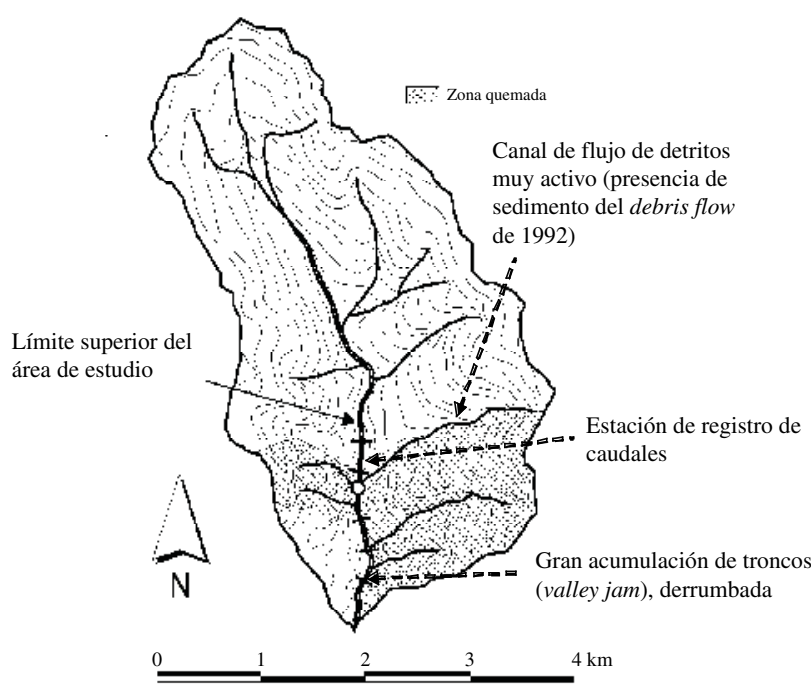

Figura 3. Esquema de la cuenca del torrente Tres Arroyos que muestra la red hidrográfica, la zona estudiada, la localización de las acumulaciones de valle (valley jams) intactas (líneas continuas transversales al canal) y del punto "nodo" (círculo blanco) en la confluencia con dos canales de flujo de detritos. También se muestra la localización de la estación de registro de caudales que funciona desde 1997 controlando $5,9 \mathrm{~km}^{2}$ de la parte alta de la cuenca.

Sketch of the study basin illustrating the channel network, the length surveyed, the location of the intact valley jams (solid lines transversal to the channel) and of the "nodal" point (white circle) at the confluence of two debris flow channels. The gauging station operating since 1997 is also shown.

clasificación de Montgomery y Buffington 1997) presenta toda la gama de tipos de estructura, desde escalón-poza (step-pool) hasta rápidos-poza (riffle-pool), con un alto grado de morfologías forzadas por la presencia del LWD. El canal es confinado, con llanura de inundación activa limitada por laderas rocosas escarpadas en los sectores más altos, mientras que es más ancho en secciones aguas abajo. Las terrazas, formadas probablemente por valleyjams como las descritas por Abbe y Montgomery (2003) en el río Queets, Washington, son comunes a lo largo del canal principal.

La hidrología y el transporte de sedimentos en el cauce del torrente Tres Arroyos han sido monitoreados desde el año 1997 (Iroumé 1997, Uyttendaele 2006). Las relaciones preliminares entre la concentración de sedimentos en suspensión, la carga de fondo y el caudal han sido determinadas por Iroumé (2003), Lenzi et al. (2004) y Uyttendaele (2006). La cuenca del torrente Tres Arroyos está afectada por procesos de transporte relacionados a flujo de detritos o flujos aluvionales de los tributarios escarpados, asociados a grandes cantidades de sedimentos y de LWD en el canal principal. Uyttendaele (2006) estima que el aluvión que ocurrió el año 1992 transportó $5.300 \mathrm{~m}^{3}$ de sedimento al cono de deyección aluvial, provenientes de un canal tributario de fuerte pendiente donde los flujos de detritos son muy frecuentes a causa de la inestabilidad natural de las márgenes (figura 3).

Intensos eventos de crecida ocurridos en los años 1972 y 1992 produjeron daños en la Ruta R-89 y en la línea férrea situados en la desembocadura de la cuenca. Testimonios de lugareños que viven cerca del cono de deyección del Tres Arroyos dan cuenta del rol crucial del LWD en la dinámica de la crecida ocurrida el año 1992, e informan que el LWD que componía el flujo aluvional fue depositado en grandes cantidades en el cono de deyección sin alcanzar la confluencia con el río Cautín. El cono de deyección se caracteriza por la presencia de grandes cantidades de LWD en distinto estado de decaimiento, con residuos leñosos dispersos sobre toda la superficie y en particular sobre las barras sin vegetación y las terrazas aluviales (figura 4). Una gran acumulación de LWD, formada por 100-150 trozos de $0,5 \mathrm{~m}$ de diámetro medio y 4-5 $\mathrm{m}$ de largo (volumen geométrico bruto de unos $600 \mathrm{~m}^{3}$, figura 5), se encuentra en la confluencia entre el canal donde se generó gran parte del flujo de detritos de la crecida de 1992 y el canal principal del Tres Arroyos (también existe otro canal más pequeño que entra en la corriente principal a unos pocos metros aguas arriba, como se ve en la figura 3 ).

Métodos de estudio. El LWD presente en el canal del estudio fue examinado en el período de marzo a abril de 2004. Todos los residuos de madera con diámetro mayor de $10 \mathrm{~cm}$ y longitud mayor de $1 \mathrm{~m}$ fueron medidos en el canal activo y en sus márgenes de inundación (floodplain) adyacentes. A cada tronco se le midió la longitud y el diámetro a través de una cinta métrica y una forcípula, con una precisión de $\pm 5 \mathrm{~cm} \mathrm{y} \pm 1 \mathrm{~cm}$, respectivamente.

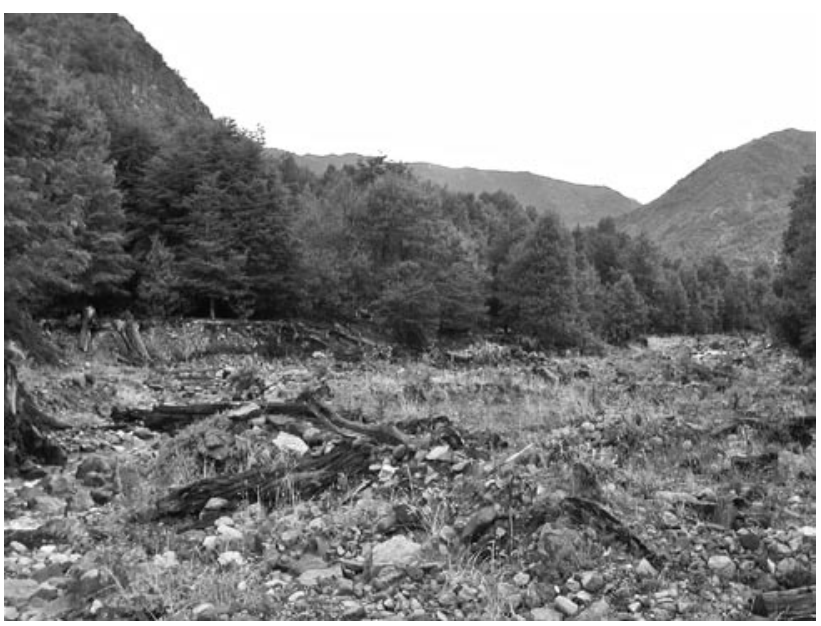

Figura 4. Vista desde aguas abajo del cono de deyección creado por el Tres Arroyos en la salida de su valle. Gran cantidad de troncos se distribuyen en la superficie del cono y se depositan entre los sedimentos.

View from downstream of the alluvial fan created by Tres Arroyos at the exit of its valley. Large quantity of LWD pieces are spread on the surface and buried in the sediments. 


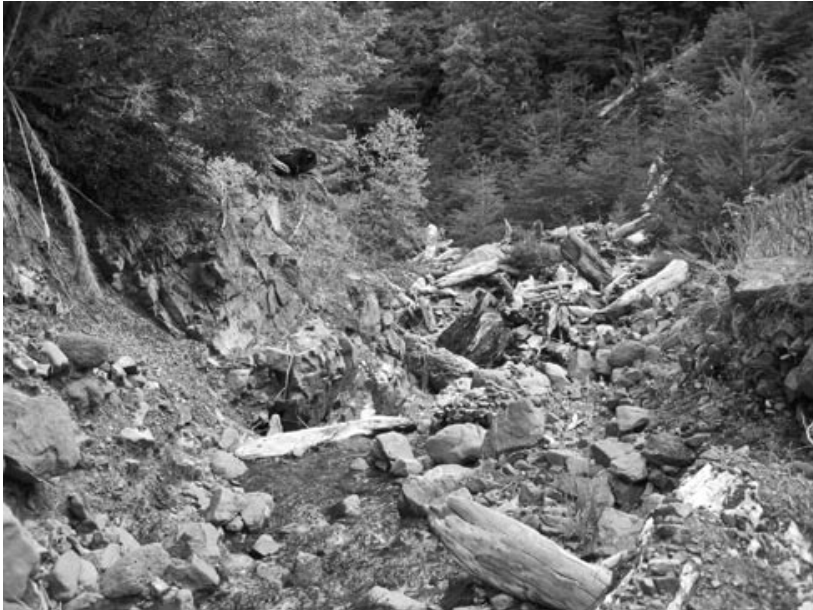

Figura 5. Vista desde aguas arriba de la gran acumulación de LWD encontrada en el punto "nodo" (véase figura 3 ).

View from upstream of the large LWD accumulation found at the "nodal" point (see figure 3 ).

Adicionalmente se midieron los trozos incluidos en los log-jams (es decir, tacos o acumulaciones de un mínimo de dos elementos), las dimensiones geométricas de los tacos (longitud, anchura y altura) y el diámetro mínimo del tronco y el diámetro máximo de las raíces de cada trozo.

El canal principal del torrente Tres Arroyos fue examinado detalladamente en un tramo de $1.540 \mathrm{~m}$ de longitud (figura 3). Adicionalmente, se recorrió un tramo de un kilómetro, desde el extremo aguas arriba del tramo anterior hasta una cascada cerca del límite inferior del bosque de araucaria. Este último segmento se caracterizaba por una morfología y una densidad de residuos leñosos similares a la parte superior del tramo examinado detalladamente. La figura 6 consta de dos imágenes representativas del canal en los dos tramos mencionados, donde se muestra el cauce aguas abajo de la confluencia (figura 6a) y el segmento superior, más estrecho, que pasa a través del bosque nativo maduro (figura $6 \mathrm{~b}$ ).

El levantamiento topográfico del perfil longitudinal (figura 7) se realizó con un distanciómetro láser con inclinómetro incorporado. Se definieron 17 tramos individuales atendiendo a la uniformidad de la pendiente, el ancho del canal y la abundancia de residuos leñosos (cuadro 2). Los tramos tienen pendientes del $3 \%$ al $15 \%$ (promedio $7 \%$ ) y un ancho a cauce lleno (bankfull) de 5,5 a 15,5 m (promedio $7,8 \mathrm{~m}$ ). El área de cuenca drenada por cada tramo, $\mathrm{A}_{\mathrm{r}}$, calculada mediante un modelo de elevación digital del terreno, varía desde 5,5 hasta $9,1 \mathrm{~km}^{2}$.

En cada tramo se midieron todos los residuos de madera (LWD), así como las siguientes características de cada uno de ellos: pendiente media del canal (s), ancho medio a cauce lleno y de las márgenes de inundación $\left(\mathrm{B}_{\mathrm{bf}} \mathrm{y}_{\mathrm{fp}}\right.$ respectivamente), profundidad media de cauce lleno $\left(\mathrm{h}_{\mathrm{bf}}\right)$, número de step $\left(\mathrm{N}_{\mathrm{st}}\right)$ y número de piedras $\left(\mathrm{N}_{\mathrm{b}}\right)$ respectivamente más altos y más grandes que $\mathrm{h}_{\mathrm{bf}}$.
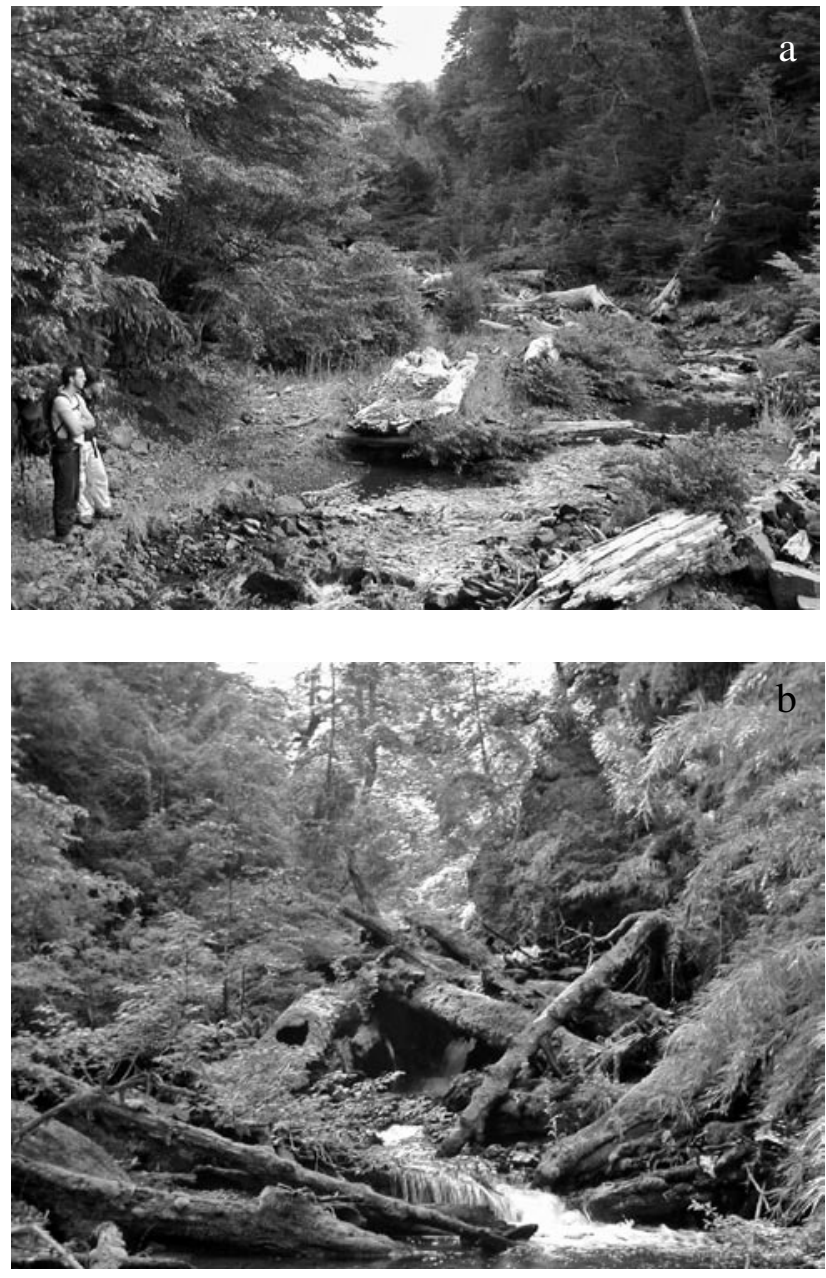

Figura 6. Vistas del canal principal desde aguas abajo: (a) aguas abajo del nodo, donde el canal y valle son más amplios, (b) aguas arriba del nodo, donde el cauce es más estrecho y los elementos más grandes de LWD se cubren con musgos densos.

Views of the main channel: above (a), downstream of the nodal point, where the channel and the valley are wider, below (b) upstream of the nodal point, where the stream is narrower and the largest LWD elements are covered with thick mosses.

En el trabajo de campo, a cada detrito leñoso se le registraron las siguientes características: tipo (tronco, raíces, tronco con raíces unidas), especie (Nothofagus/ araucaria/conífera), la orientación con respecto al flujo (paralelo, ortogonal, oblicuo), el estado de decaimiento definido a través de la observación de los trozos de madera en tres niveles (bajo, cuando se notaba presencia de corteza y hojas, secas o no; medio, no había presencia de hojas ni corteza, pero la madera no se notaba porosa ni se deshacía, y alto con presencia hasta de ataques de hongos o de insectos y cuando la madera estaba muy porosa y se deshacía fácilmente), el origen (erosión de márgenes, deslizamiento de las laderas adyacentes, mortalidad natural, transportado por la corriente) y la posición (log-step, en el canal, en el canal a cauce lleno, 
suspendido formando un puente sobre el canal, en las márgenes inundables). Los trozos de madera calificados como "en el canal" fueron aquellos que se encontraban a una elevación más baja que la altura de cauce lleno, a excepción de los escalones de troncos (log-step) que

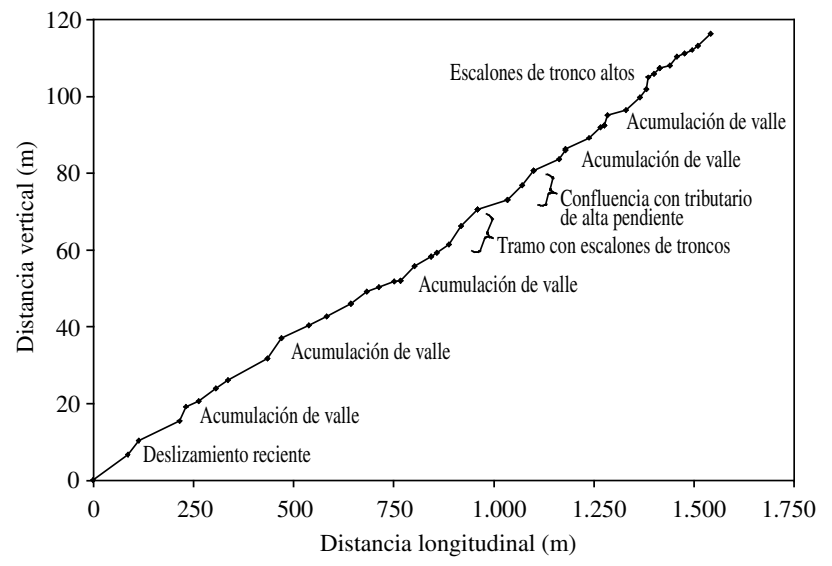

Figura 7. Perfil longitudinal del tramo examinado del canal donde se muestran las principales características del LWD y otros procesos externos.

Longitudinal profile of the surveyed channel length, showing the main LWD-related features and other external forcing. forman una categoría diferente. En relación a los escalones de troncos se midió también la altura del desnivel y la profundidad de las pozas al pie de los escalones. Los elementos de LWD encontrados a una altura correspondiente al nivel de cauce lleno fueron considerados como un grupo separado.

Los elementos calificados como "suspendidos formando un puente sobre el cauce" fueron aquellos ubicados a una elevación superior al nivel de cauce lleno. En cambio, los troncos situados por encima del nivel de cauce lleno pero en el área adyacente sujeta a inundaciones de baja frecuencia fueron considerados como elementos en los márgenes del canal. En el caso de troncos largos que ocupaban diversas porciones del canal, la localización asignada correspondió al lugar donde se emplazaba la mayor parte del tronco; a su vez, a los trozos de madera ubicados en parte o por completo sobre el nivel máximo de inundación no se les estimó el volumen parcial del tronco dentro del nivel del flujo, registrándose sólo la longitud total del mismo. Este procedimiento pudo producir una leve sobreestimación del volumen total de LWD ubicados en el cauce del torrente.

El volumen de sedimento depositado aguas arriba de los escalones de troncos y valley-jams se calculó considerando una cuña sólida de dimensiones geométricas (longitud, ancho aguas arriba y aguas abajo, altura) medidas con

Cuadro 2. Características de los 17 tramos estudiados en el torrente Tres Arroyos.

Characteristics of the 17 investigated reaches of Tres Arroyos torrent.

\begin{tabular}{|c|c|c|c|c|c|c|c|c|}
\hline Tramo & $\begin{array}{l}\text { Longitud } \\
\qquad(\mathrm{m})\end{array}$ & $\begin{array}{l}\text { Pendiente } \\
(\mathrm{m} / \mathrm{m})\end{array}$ & $\begin{array}{l}\text { Ancho de } \\
\text { cauce lleno } \\
\text { (m) }\end{array}$ & $\begin{array}{l}\text { Ancho de la } \\
\text { llanura de } \\
\text { inundación } \\
\text { (m) }\end{array}$ & $\begin{array}{l}\text { Morfología } \\
\text { del canal* }\end{array}$ & $\begin{array}{c}\text { Tipo de } \\
\text { bosque } \\
\text { adyacente }^{* *}\end{array}$ & Notas & $\begin{array}{l}\text { Características } \\
\text { de los LWD*** }\end{array}$ \\
\hline 1 & 82 & 0,08 & 7,6 & 17,3 & SP, CA & $\mathrm{JN}, \mathrm{CP}$ & - & DD roto \\
\hline 2 & 32 & 0,11 & 9,1 & 16,7 & $\mathrm{CA}$ & $\mathrm{JN}, \mathrm{CP}$ & Deslizamientos recientes & Tacos, DD roto \\
\hline 3 & 117 & 0,08 & 8,2 & 11,6 & SP, CA & $\mathrm{JN}, \mathrm{CP}$ & Deslizamientos & DD \\
\hline 4 & 106 & 0,07 & 7,1 & 21,3 & SP, RA & $\mathrm{JN}, \mathrm{PC}$ & $\begin{array}{l}\text { Deslizamientos, confluencia } \\
\text { con tributario a alta pendiente }\end{array}$ & Tacos, DD roto \\
\hline 5 & 134 & 0,07 & 5,8 & 27,3 & SP, RA & $\mathrm{JN}, \mathrm{PC}$ & - & Escalones, tacos \\
\hline 6 & 69 & 0,05 & 7,6 & 30,2 & RA & $\mathrm{JN}, \mathrm{PC}$ & Cono tributario & $\mathrm{DD}, \mathrm{DD}$ roto, tacos \\
\hline 7 & 105 & 0,05 & 7,1 & 23,8 & RA, CA & $\mathrm{JN}, \mathrm{PC}$ & Terrazas anchas & - \\
\hline 8 & 124 & 0,05 & 7,2 & 20,4 & $\mathrm{CA}, \mathrm{SP}$ & $\mathrm{JN}, \mathrm{PC}$ & Terrazas anchas & Curva, DD roto \\
\hline 9 & 76 & 0,08 & 7,8 & 20,5 & SP, RA & VN, JN & Erosión de las márgenes & $\mathrm{DD}$ \\
\hline 10 & 116 & 0,11 & 8,0 & 20,0 & SP & VN, JN & $\begin{array}{l}\text { Confluencia con subcuenca } \\
\text { desestabilizada }\end{array}$ & $\begin{array}{l}\text { Escalones, canal } \\
\text { abandonado }\end{array}$ \\
\hline 11 & 74 & 0,03 & 15,5 & 22,0 & RA & $\mathrm{VN}, \mathrm{PC}$ & Área amplia de deposición & DD roto \\
\hline 12 & 65 & 0,12 & 8,0 & 16,0 & SP, CA & $\mathrm{PC}$ & $\begin{array}{l}\text { Confluencia con dos canales } \\
\text { de debris flow }\end{array}$ & $\begin{array}{l}\text { Escalones, pila enorme } \\
\text { de LWD }\end{array}$ \\
\hline 13 & 79 & 0,06 & 6,8 & 17,7 & SP, RA & $\mathrm{VN}$ & - & Escalones, DD \\
\hline 14 & 97 & 0,07 & 5,6 & 8,0 & SP, CA & VN & Lecho en rocas & Taco \\
\hline 15 & 90 & 0,08 & 6,2 & 13,7 & RA & $\mathrm{VN}$ & Erosión de las márgenes & $\mathrm{DD}$ \\
\hline 16 & 49 & 0,15 & 5,5 & 8,0 & SP & $\mathrm{VN}$ & Cono tributario & Escalones altos, DD roto \\
\hline 17 & 128 & 0,07 & 7,0 & 11,7 & SP, RA & VN & Deslizamientos & Escalones, tacos \\
\hline
\end{tabular}

* $\quad \mathrm{SP}=$ secuencia escalón-poza ( (step-pool), $\mathrm{CA}=$ cascada, $\mathrm{RA}=$ rápido.

** $\mathrm{JN}=$ renoval de Nothofagus, $\mathrm{PC}=$ plantación de coníferas, $\mathrm{VN}=$ Nothofagus adulto.

*** $\mathrm{DD}=$ dique de detritos (debris dam - valley jam). 
una cinta, sin considerar la porosidad. El volumen de los trozos de madera se calculó por referencia al diámetro, $\mathrm{D}_{\mathrm{log}}$, medido en la mitad de su longitud, y a la longitud, $\mathrm{L}_{\mathrm{log}}$, asimilándolo al volumen de un cilindro, como se hace comúnmente para estudios sobre el LWD. El volumen de las raíces se aproximó al volumen de un cilindro de diámetro igual al del tronco junto a la raíz por la altura de las raíces.

\section{RESULTADOS}

Volumen de LWD, densidad espacial y características. El volumen medio de LWD almacenado en el área del canal del torrente Tres Arroyos, incluyendo las márgenes inundables, es extremadamente alto. Sobre la base de la longitud del tramo de canal estudiado, dicho volumen se estimó en $1.198 \mathrm{~m}^{3} / \mathrm{km}$ de LWD, correspondientes a $1.530 \mathrm{~m}^{3} / \mathrm{ha}$ del área de cauce lleno. El número medio de elementos por kilómetro de canal fue de $1.550 \mathrm{y}$ de 1.979 elementos por hectárea de área del cauce lleno. Si sólo se consideran los elementos dentro del cauce lleno, el volumen alcanza a $556 \mathrm{~m}^{3} / \mathrm{km}$ o $710 \mathrm{~m}^{3} / \mathrm{ha}$, mientras que la densidad espacial media de los elementos fue de 786/km o 1.004/ha.

Las dimensiones de los elementos de LWD eran de hasta $2,2 \mathrm{~m}$ de diámetro y $26 \mathrm{~m}$ en longitud. El tamaño medio de los elementos resultó de $0,41 \mathrm{~m}$ de diámetro y $3,25 \mathrm{~m}$ de largo en toda la población de LWD examinada, mientras que los elementos presentes en el área del cauce lleno tendieron a ser levemente más grandes, de $0,50 \mathrm{~m}$ y 3,00 m, respectivamente. La proporción numérica entre elementos de Nothofagus spp. y Araucaria araucana fue la misma tanto al incluir los elementos "marginales" (es decir, los que están sobre la llanura de inundación y no en el canal de bankfull) como al no incluirlos: $94 \%$ y $6 \%$, respectivamente. En el canal se encontraron muy pocos trozos de madera de coníferas provenientes de las plantaciones y su número no influyó en la caracterización de los LWD en el tramo estudiado. Sólo un 2\% de los elementos presentaba un grado de decaimiento bajo (hojas o corteza todavía pegadas), mientras que el $27 \%$ y $15 \%$ de los elementos dentro del área activa total del canal (cauce y márgenes inundables) y del cauce lleno, respectivamente, mostraba un grado de descomposición alto.

Aproximadamente dos tercios de los elementos de LWD fueron encontrados en tacos o acumulaciones, y solamente un tercio como elementos individuales. No hay grandes diferencias entre el área activa total del canal y del cauce lleno: el número de elementos categorizados como "formando tacos o acumulaciones" fue muy similar en ambas situaciones, $66 \%$ y $68 \%$, respectivamente. De la misma forma, no hubo discrepancias evidentes entre el área activa total del canal y el área correspondiente al cauce lleno con respecto al tipo de elemento de LWD: solamente el $1 \%$ fueron raíces, $3-4 \%$ troncos con raíces, y
95-96\% troncos sin raíces. La orientación de los elementos en relación a la dirección del flujo se distribuyó casi en la misma proporción entre categorías oblicuas, ortogonal y paralelas, con una débil dominancia (el 37\%) de los elementos en orientación oblicua.

Observando el posible origen del LWD, en el $88 \%$ de los elementos dentro del cauce lleno (el $83 \%$ dentro del área total del canal) se encontraron evidencias de transporte por el río, el 10\% (13\%) fue asociado a caídas naturales de árboles, y el restante a deslizamientos y erosión de laderas. El ingreso de residuos leñosos al canal principal desde canales de flujo de detritos laterales se incluyó en la categoría "transportado". Los elementos de madera en el torrente Tres Arroyos resultaron bastante grandes en comparación con las dimensiones del canal. El cociente entre la longitud de cada elemento y el ancho del cauce lleno y entre el diámetro de cada elemento y la profundidad media de cauce lleno $\left(\mathrm{h}_{\mathrm{bf}}\right)$ fue en promedio de 0,46 y 0,98 , respectivamente. A pesar de las grandes dimensiones de los elementos, se evidencia un transporte absolutamente activo de LWD en el flujo, de acuerdo al alto grado de acumulación, la escasa presencia de troncos con raíces y la existencia de grandes elementos de Araucaria araucana y Nothofagus spp. aguas abajo de sus posibles localizaciones originales.

Dimensiones y características de los tacos o acumulaciones. En los tramos examinados se encontró un total de 123 tacos o acumulaciones de elementos de LWD en el área del canal (cauce y márgenes inundables), que corresponden a una densidad espacial de 80 tacos por kilómetro de longitud de canal. Si se consideran sólo los tacos encontrados por lo menos en parte dentro del cauce lleno que pueden afectar la morfología del cauce mismo, el número total de acumulaciones desciende a 78, correspondiente a 51 tacos por kilómetro de longitud de canal, con un espaciamiento medio entre tacos de cerca de $20 \mathrm{~m}$. En cuanto al volumen de LWD, la mitad del volumen total de madera almacenado en tacos corresponde a acumulaciones situadas fuera del cauce.

Los tacos o acumulaciones en Tres Arroyos estaban formados, en promedio, por 13 troncos (rango de 2 a 93 elementos), mientras que el volumen medio de madera contenido en los mismos era de $9 \mathrm{~m}^{3}$ (entre 0,2 y $68 \mathrm{~m}^{3}$ ). El diámetro y la longitud media de los elementos en las acumulaciones eran de $0,4 \mathrm{~m}$ y $3,0 \mathrm{~m}$, respectivamente, similares a las dimensiones medias de todos los elementos de madera encontrados en el cauce. Las dimensiones geométricas medias de los tacos eran: altura $1,1 \mathrm{~m}$, longitud $6,2 \mathrm{~m}$ y ancho 4,2 $\mathrm{m}$. El volumen geométrico promedio de las acumulaciones, calculado suponiendo una forma de paralelepípedo, es de $45 \mathrm{~m}^{3}$ (rango: 0,8-315 $\mathrm{m}^{3}$ ), con un cociente medio entre el volumen de madera y el volumen de la acumulación, de aproximadamente 0,2.

Analizando el origen de los elementos de LWD encontrados en los tacos o acumulaciones, la gran mayoría 
parece haber sido transportada desde aguas arriba (el 86\%). Los elementos claves (key pieces) derivaron de los deslizamientos (4\%), de la erosión de las márgenes (1\%) y de la mortalidad natural (9\%) de los árboles que crecen en las cercanías del curso de agua. Esta distribución se asemeja a la representada en la figura 8 para la población total de los elementos de LWD (individuales y en acumulaciones). Por el contrario, dentro de los tacos dominaba la orientación oblicua (42\%), con pocos elementos ordenados ortogonalmente (29\%) a la dirección de la corriente.

Se observaron diversos tipos de log-jams (siguiendo la clasificación de Abbe y Montgomery 2003) en el torrente Tres Arroyos, variando de in situ a "combinación" y a "tacos" o acumulaciones producidas por transporte de trozos por crecidas del caudal (figura 8). Los tipos in situ y de combinación estuvieron representados principalmente por los escalones de troncos y las acumulaciones o tacos de valle (o grandes debris-dam), que serán analizados detalladamente en las secciones siguientes. El tipo de transporte dominante de acumulaciones se refiere a aquellos que se alinean entre los bordes del canal a cauce lleno y la línea de máxima ocupación de las llanuras inundables (bankfull bench jams), con poca presencia de acumulaciones o tacos inestables situados en las márgenes de inundación (depósitos de banco y los revestimientos). En Tres Arroyos se observaron pocos tacos generados por aluviones o flujo de detritos, pero los existentes correspondían a las acumulaciones más grandes, situadas mayoritariamente fuera del canal, en las confluencias con los tributarios de alta pendiente (cuadro 2, figuras 5 y 9).

Posición y rol morfológico de LWD en el torrente Tres Arroyos. Con respecto a la llanura de inundación (floodplain), un poco más de la mitad de los troncos de LWD medidos en el Tres Arroyos se encontraba fuera del área de cauce lleno (figura 10), y estaban dispuestos como acumulaciones de residuos ubicados en las desembocaduras de los tributarios, formados por elementos aislados y tacos inestables depositados durante el apogeo de las crecidas a lo largo del canal principal, o tacos estables en el valle. No se aprecian diferencias al comparar la posición de todos los elementos de madera (individualmente o en acumulaciones, figura 10a) con la de los elementos individuales (figura 10b). Los tacos inestables o los elementos únicos depositados en las márgenes del canal tienen una baja influencia en la morfología del canal o de la llanura de inundación (Abbe y Montgomery 2003), aunque alteran la localización de la vegetación en esta última, limitan la degradación del fondo y promueven el depósito de sedimento, disminuyendo así la profundidad del canal (Kochel et al. 1987). Por otra parte, los elementos de LWD estabilizados en las márgenes del canal formaban parte de estructuras complejas que bloqueaban el canal y se extendían a la llanura de inundación (es decir, valley jams) o indicaban la existencia previa de estructuras similares, ahora derrumbadas (véase una mayor descripción más adelante en el texto), con las terrazas

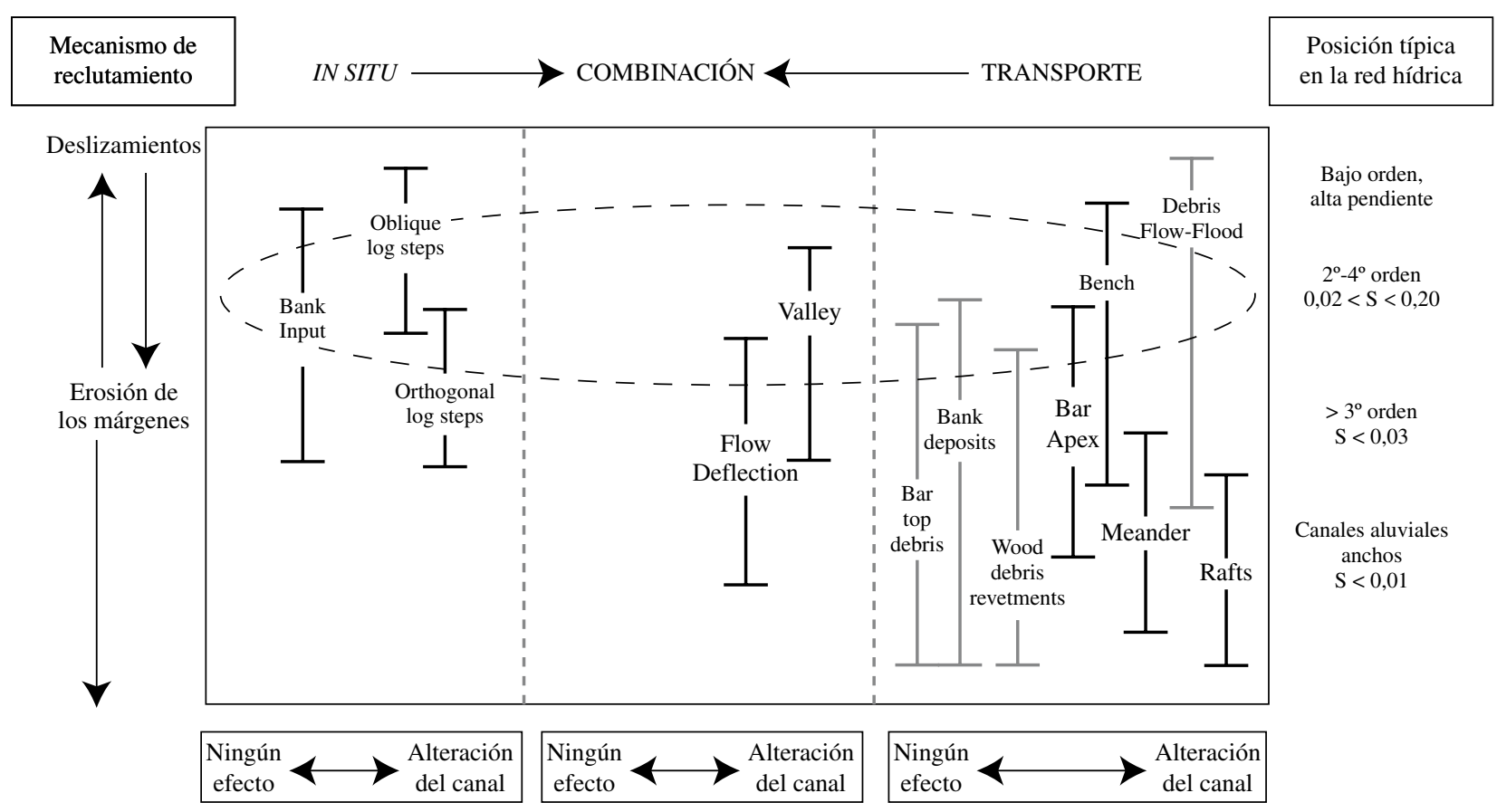

Figura 8. Los tipos de acumulaciones de troncos encontrados en el torrente Tres Arroyos (línea discontinua), según la clasificación de Abbe y Montgomery (2003).

The types of $\log$ jams found in Tres Arroyos (dashed line) according to the classification by Abbe and Montgomery (2003). 
algunos metros más altas que la llanura de inundación activa actual.

El volumen de LWD registrado en el área de cauce lleno (bankfull) estaba distribuido, en partes iguales, como trozos dispersos sobre el fondo del canal y alineados a los bordes de éste (figura 10). Muy pocos elementos constituían escalones de troncos o se encontraban suspendidos formando un puente sobre el canal. Los elementos a nivel de cauce

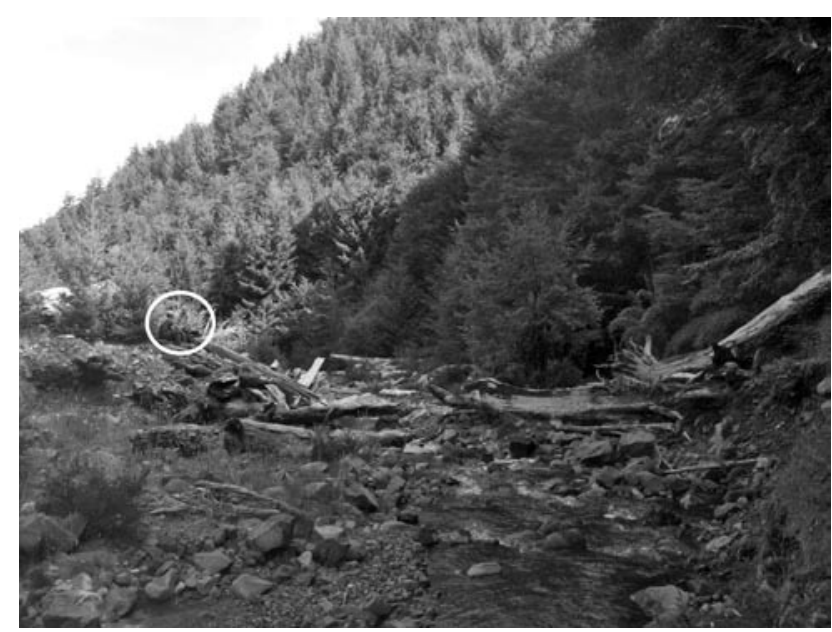

Figura 9. Vista desde aguas arriba de la gran acumulación de valle derrumbada (el indicado en figura 3). Como escala se observan dos personas. La gran cantidad de sedimento que estuvo acumulado en el pasado detrás de esta estructura se puede deducir de la altura de las capas de sedimento a la izquierda de la figura.

View from upstream of the large collapsed valley jam (see location on figure 3). For scaling, two persons are circled. The large amount of sediment once stored behind this LWD structure can be inferred from the sediment layers on the left of the picture. lleno, particularmente los orientados en dirección paralela al flujo, no parecían influir mayormente en la morfología del cauce sino que ayudaban a estabilizar las márgenes y protegerlas de la acción directa del flujo principal, limitando así la erosión de las mismas en tanto los LWD no cambien de lugar. Muy raramente se observaron deformaciones del fondo del cauce (pozas-barras) vinculadas directamente a la presencia de LWD, sugiriendo que la mayoría de estos residuos estaba en movimiento o no había tenido aún tiempo de producir modificaciones importantes en la morfología del lecho fluvial desde su depósito durante la última crecida.

Aunque los elementos que formaban escalones de troncos (log-steps) representan apenas una pequeña proporción de la población total de LWD, éstos, junto con las acumulaciones de valle (valley jams), ejercían una influencia importante en la morfología del canal y la disipación energética del flujo en el Tres Arroyos (cuadro 3). Las dimensiones medias de los elementos de LWD que formaban el umbral del escalón fueron 0,47 m de diámetro y $6,11 \mathrm{~m}$ de longitud. La mayoría de los escalones de troncos se encontraron dispuestos ortogonalmente a la dirección de la corriente $(85 \%)$, con evidencias de un cierto transporte fluvial (82\%). La porción restante parecía haberse generado por la mortalidad natural de los árboles sobre las márgenes y laderas adyacentes, formando los escalones más altos. Se identificaron veintisiete escalones de troncos, sin contar los que forman parte de acumulaciones de valle, que correspondían a una densidad media de 1,7 log-step por 100 $\mathrm{m}$ de la longitud del canal. El número total de escalones (clast-steps, log-steps y mezclados) a lo largo de la sección examinada del cauce fue de 122 , con una proporción de escalones de troncos de alrededor del $22 \%$.

La altura media de desnivel de los escalones de troncos era de $0,87 \mathrm{~m}$, y la pérdida acumulada de elevación debida a ellos de $2,5 \mathrm{~m}$, lo que representaba el $19 \%$ de
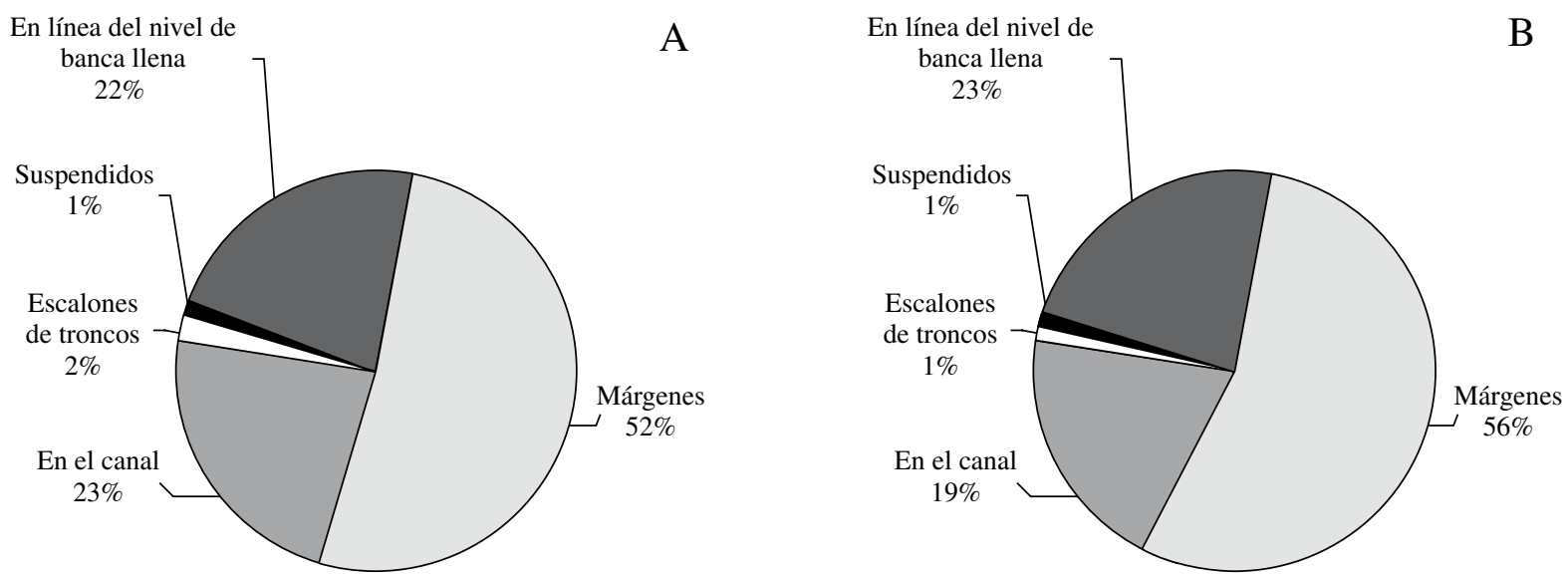

Figura 10. Posición de los elementos en el torrente Tres Arroyos considerando los elementos individuales dispersos más los sobrepuestos en acumulaciones (A) y sólo los elementos individuales (B).

Distribution of LWD elements based on their location (see text for definition of the categories). (A) for all the LWD pieces, i.e. both single and jam-forming; (B) for single elements only. 
Cuadro 3. Características medias de los escalones de troncos (log-steps) y de las acumulaciones de valle (valley jams) en el torrente Tres Arroyos. Los valores entre paréntesis representan el rango de la variación de los parámetros.

Mean characteristics of log-steps and valley jams in Tres Arroyos. Figures in brackets represent the range of variation of the parameters.

\begin{tabular}{lcc}
\hline Característica & $\begin{array}{c}\text { Escalones } \\
\text { de troncos } \\
(\text { Log-steps })\end{array}$ & $\begin{array}{c}\text { Acumulaciones } \\
\text { de valle } \\
\text { (Valley jams) }\end{array}$ \\
\hline Frecuencia & 1,7 cada 100 m & 0,33 cada 100 m \\
Desnivel (m) & $0,87(0,15-3,00)$ & $2(1,5-2,7)$ \\
Pérdida acumulada de & 19 & 8 \\
elevación & & \\
(\% del total) & $37(0,5-175)$ & $191(52-308)$ \\
Sedimento depositado $\left(\mathrm{m}^{3}\right)$ & $0,47(0,20-1,10)$ & $0,36(0,10-1,00)$ \\
Diámetro del LWD $(\mathrm{m})$ & $6,11(1,25-25,70)$ & $3,20(1,00-12,00)$ \\
Longitud del LWD $(\mathrm{m})$ & $2,13(0,09-24,41)$ & $0,56(0,01-6,81)$ \\
Volumen del LWD $\left(\mathrm{m}^{3}\right)$ & & \\
\hline
\end{tabular}

la pérdida total de elevación en la sección examinada del cauce. El volumen de la cuña de sedimento detrás de los escalones de troncos (volumen sólido dentro del cauce lleno) alcanzaba de 0,5 hasta $175 \mathrm{~m}^{3}$, con un volumen total de aproximadamente $1.000 \mathrm{~m}^{3}$.

Con respecto a las acumulaciones de valle (valley jams, large debris-dams), en el torrente Tres Arroyos se observaron cinco grandes diques de residuos (debris dams) intactos que se extendían a través del canal y las márgenes (valley jams, según Abbe y Montgomery 2003), y siete más pero que se encontraron derrumbados durante el estudio, todos asociados a terrazas con troncos clave ubicados ortogonalmente que sobresalían fuera de las márgenes como elementos acumulados sueltos (loose) y apilados (racked). La localización de estos cinco diques intactos se puede apreciar en la figura 3 , donde se indican con una línea continua transversal al canal. La acumulación más grande de valle (valley jam) derrumbada (ver figura 9) se estimó de 3,5 m de alto, $22 \mathrm{~m}$ de ancho y con un depósito de aproximadamente $1.700 \mathrm{~m}^{3}$ de sedimento (volumen sólido de la cuña), y había causado el ensanchamiento del lecho del canal hasta $60 \mathrm{~m}$ aguas arriba. Esta estructura derrumbada estaba situada entre los tramos 6 y 7 (cuadro 2), algunos metros agua arriba de un dique de residuos intacto ubicado en la confluencia con un tributario de alta pendiente, el que probablemente entrega enormes cantidades de LWD. Aguas abajo de este tributario se encontró un gran cono de deyección producido por antiguos debrisflow, los que probablemente ocurrieron más frecuentemente después del incendio (figura 4), afectando la morfología del canal principal. Además, el tramo 7 (situado aguas arriba del dique de residuos derrumbado) se caracterizó por amplias terrazas escalonadas con árboles de diferentes edades, probablemente ligadas a la evolución del cono de deyección del tributario y a las acumulaciones de valle. La confluencia múltiple de los dos canales de flujo de detritos (el punto "nodo" marcado en figura 3), donde se encontró una gran acumulación de LWD descrita en la sección anterior, fue probablemente la localización de un gran dique de residuos, dado que existe un depósito de al menos $3 \mathrm{~m}$ de sedimentos, del cual sobresalen hacia el cauce algunos troncos que fueron enterrados durante aluviones antiguos (figura 5).

Las acumulaciones de valle intactas tenían una altura promedio de $2 \mathrm{~m}$ (rango 1,5-2,7 $\mathrm{m}$ ) con un desnivel acumulado de $10 \mathrm{~m}$. Sumando a este valor el desnivel acumulado debido a los escalones de troncos $(23,5 \mathrm{~m})$, la pérdida total de la elevación debido a los LWD era de $33,5 \mathrm{~m}$, correspondiente a una disipación del $27 \%$ de la energía potencial de la corriente en la sección examinada del canal (cuadro 3).

El volumen de sedimento acumulado aguas arriba de estas presas de residuos varía de 52 hasta $308 \mathrm{~m}^{3}$ (volumen sólido de la cuña), con un promedio de $191 \mathrm{~m}^{3}$ y un total de $956 \mathrm{~m}^{3}$. El valor total resultó casi igual al volumen acumulado del sedimento atrapado detrás de los log-steps $\left(1.000 \mathrm{~m}^{3}\right)$. Es así como el total de sedimentos acumulados en el canal principal debido a los LWD era de $2.000 \mathrm{~m}^{3}$, correspondiente aproximadamente al $150 \%$ de la producción anual del sedimento de la cuenca. Sin embargo, en este valor no se incluyeron algunos depósitos más pequeños dentro del canal, estabilizados por elementos de madera y el sedimento almacenado en la llanura de inundación, de modo que la cantidad total de sedimento retenida en el cauce debido a los LWD puede ser mucho más alta.

Las acumulaciones de valle en el torrente Tres Arroyos estaban formadas por elementos de madera, transportados desde aguas arriba, cuyo número variaba entre 17 y 63 . Las dimensiones medias de los elementos de LWD en los diques eran $0,36 \mathrm{~m}$ de diámetro y 3,20 $\mathrm{m}$ de longitud, las cuales resultaron similares a las dimensiones medias de todos los elementos de LWD en el torrente, i.e., de 0,41 y 3,25 m. Los elementos más grandes dentro de cada dique, representados por los troncos más estables (key-pieces), presentaban diámetros entre 0,8 y $1,0 \mathrm{~m}$, y longitudes entre 6 y $12 \mathrm{~m}$. Estos elementos provenían fundamentalmente de la erosión de las márgenes, mortalidad natural de los árboles o del flujo de detritos. El volumen total de madera contenido en un dique en promedio de 19,6 $\mathrm{m}^{3}$ (rango 9,7-33,0 $\mathrm{m}^{3}$ ). La distancia media entre las cinco acumulaciones de valle fue aproximadamente de $300 \mathrm{~m}$ (tres acumulaciones por kilómetro de longitud de canal), con un espaciamiento aleatorio (figuras 3 y 7).

Acumulaciones de valle con similares espaciamientos se observaron aguas arriba de la sección examinada, donde el canal se encuentra marginado por bosque nativo maduro, similar al observado entre el tramo 13 y el 17 (cuadro 2, figura 6). A lo largo de esta sección superior del cauce, el aporte de residuos leñosos debido a debris flow pareció 
menos importante, mientras que los afloramientos de roca que estrechan el canal representaban localizaciones favorables para el establecimiento de acumulaciones de valle, posiblemente con elementos clave más pequeños.

Otros tipos de influencia morfológica de los LWD, como acumulaciones que desvían el flujo, según lo indicado en la figura 9, se podrían también esperar en canales relativamente escarpados de orden inferior, como el torrente Tres Arroyos (Abbe y Montgomery 2003). De hecho, se observaron algunos ejemplos de tales acumulaciones. Sin embargo, su impacto en la morfología del canal parecía limitado, con excepción de una curva inusualmente cerrada (tramo 8), y una acumulación de troncos causado por un aluvión, el que dejó un canal abandonado de $30 \mathrm{~m}$ de largo al lado del actual curso de agua (tramo 10).

\section{DISCUSIÓN}

Volumen de LWD, densidad espacial y características. Los valores de volumen medio de LWD en relación a la longitud del canal o al área del cauce lleno resultan muy altos en Tres Arroyos, sólo comparables con aquellos encontrados en bosques nativos maduros de pino oregón y secuoyas (Sequoia sempervirens (Lamb. ex D. Don) Endl.) en el noroeste de Norteamérica, donde se encuentra con frecuencia $1.000 \mathrm{~m}^{3} /$ ha de madera (Gurnell et al. 2002, Gurnell 2003).

La mayor parte del canal examinado (tramos desde 1 hasta 12) es adyacente al área afectada por el incendio en el último siglo (figura 4). Se podría entonces haber esperado una densidad distinta de LWD (ver Zelt y Wohl 2004) entre estas secciones y los tramos localizados aguas arriba (13-17). Una comparación (con la prueba U de Mann-Whitney) de los valores medidos de residuos leñosos en estos tramos no revela diferencia estadística $(P>$ $0,05)$ cuando se consideran todos los elementos de LWD en el área total del canal. Sin embargo, si se consideran solamente los troncos ubicados dentro del cauce lleno, los tramos aguas arriba presentan, en promedio, dos veces más volumen de LWD que los tramos aguas abajo adyacentes a las áreas afectadas por el incendio (diferencia estadísticamente significativa a un valor de $P<0,05)$. Tal resultado no se puede atribuir sólo al efecto del incendio, ya que también hay diferencias en las características geomorfológicas, tales como el ancho del valle y el gradiente de las vertientes entre estos dos sectores.

Dimensiones y características de los tacos o acumulaciones. Los valores determinados en Tres Arroyos respecto a número y densidad espacial de acumulaciones o tacos formados por LWD son similares al espaciamiento medio de las acumulaciones encontrado por Gurnell et al. (2002) en un curso de agua de segundo orden en el Reino Unido y al número medio de acumulaciones por unidad de longitud de canal descrito para el río Queets por Abbe y
Montgomery (2003), mientras que son generalmente más pequeños que los valores $(25-50 \mathrm{~m}$ ) encontrados por Martin y Benda (2001) para áreas de tamaño similar (5-10 km²) en la cuenca del Game Creek, Alaska. Sin embargo, Martin y Benda (2001) definieron como taco o acumulación sólo los que obstruían al menos el $20 \%$ del ancho del cauce lleno. En Tres Arroyos, sólo 14 acumulaciones de trozos alcanzaban a reducir el ancho de la sección transversal, con un porcentaje de bloqueo medio del $40 \%$.

La distribución de los tipos de acumulaciones en el Tres Arroyos es muy similar a lo descrito por Abbe y Montgomery (2003) en torrentes de alta pendiente (canales de segundo-cuarto orden, con pendientes de 0,02 ) del río Queets. Esto refleja la abundancia de árboles grandes en el bosque adyacente que entrega al canal una cantidad importante de trozos de madera de gran tamaño y por ende muy estables, y fragmentos más pequeños capaces de acumularse en grandes tacos de valle o como acumulaciones más inestables en los bordes del canal y de las márgenes.

Posición y rol morfológico de LWD en el torrente Tres Arroyos. En la costa noreste de Norteamérica, los escalones de troncos parecen ser más frecuentes que en el torrente Tres Arroyos. Faustini y Jones (2003) en el Mack Creek, un canal de tercer orden en Oregon, registraron cerca 1,7 log-steps por cada 100 metros, mientras que en el río Queets, en cuencas con superficies menores a 10 $\mathrm{km}^{2}$, Montgomery et al. (2003) registraron entre 5 y 10 escalones de troncos por cada $100 \mathrm{~m}$. En Alaska, en cauces que drenan cuencas de 0,12 a $0,35 \mathrm{~km}^{2}$ de superficie, Gomi et al. (2003) registraron hasta 35 escalones por 100 $\mathrm{m}$, formados por trozos leñosos grandes o pequeños, y el $51 \%$ de los escalones formados por troncos. Un porcentaje similar $(45 \%)$ de escalones formados sólo por troncos (LWD) fue registrado por Curran y Wohl (2003) en una cuenca con una superficie inferior a $10 \mathrm{~km}^{2}$ en Cascade Range (Washington, Estados Unidos). Jackson y Sturm (2002) encuentran que la importancia de los escalones "orgánicos" puede ser incluso más alta, ya que en cursos de agua de primer y segundo orden en la Coast Range (Washington, Estados Unidos) los steps "inorgánicos" representan sólo el 19\% del número total de escalones. Previamente, Wohl et al. (1997) correlacionaron el porcentaje de escalones formados por LWD con el tamaño de la cuenca/canal en los cauces de Montana (Estados Unidos), registrando valores desde 50\% (área 1-2 $\mathrm{km}^{2}$ ) hasta $10 \%$ (área $>6 \mathrm{~km}^{2}$ ). Por el contrario, frecuencias más pequeñas de escalones de troncos fueron registradas por Marston (1982) en canales de tercer orden en la Oregon Coast Range, con un valor de 0,4 escalones por $100 \mathrm{~m}$, estimando una disipación potencial de la energía del 6\%. Diversos autores han estimado tasas de disipación de energía muy altas (10-80\%, y hasta 100\%), causadas por las acumulaciones de troncos, en diversas regiones de los Estados Unidos (Heede 1972, Swanson 
et al. 1976, Bilby 1979, Keller y Swanson 1979, Keller y Tally 1979, Faustini y Jones 2003).

Como referencia, Lenzi et al. (2004) estimaron la producción anual media de sedimento para Tres Arroyos en la estación fluviométrica (figura 3) en 3.564 t/año, valor que corresponde, si se asume una masa específica del sedimento de $2,7 \mathrm{t} / \mathrm{m}^{3}$, a $1.320 \mathrm{~m}^{3} /$ año. Por lo tanto, los escalones de troncos en Tres Arroyos eran responsables de un depósito de sedimento en el canal equivalente a cerca del $75 \%$ de la producción anual media de sedimento de la cuenca. Para estimar una proporción más exacta habría sido necesario medir la porosidad de los depósitos de sedimento, lo que no se hizo.

En cuanto al depósito de sedimentos, Marston (1982) estimó que el sedimento acumulado aguas arriba de acumulaciones de troncos en los ríos de Oregon era de alrededor del $123 \%$ de la producción anual media de sedimento. Sin embargo, otros autores postulan que los LWD crean sitios de depósito capaces de acumular volúmenes desde 10 hasta 15 veces la producción anual de sedimento en ríos de montaña (Mehagan 1982, Swanson y Fredriksen 1982).

En el torrente Tres Arroyos el total de sedimentos acumulados en el canal principal debido a los LWD corresponde aproximadamente al $150 \%$ de la producción anual del sedimento de la cuenca, cercano al valor encontrado por Marston (1982).

El volumen y distancia media entre las acumulaciones de troncos en situación de valle, en el torrente Tres Arroyos, son similares a lo encontrado en el Queets River en áreas de drenaje entre 5 y $10 \mathrm{~km}^{2}$ (véase figura 11 en Abbe y Montgomery 2003). Sin embargo, el tamaño de estas acumulaciones es demasiado pequeño como para analizar su distancia desde una perspectiva geoestadística, tal como lo hacen recientemente Wing et al. (1999), Keim et al. (2000) y Kraft y Warren (2003). La localización de las acumulaciones de valle está claramente asociada (cuadro 2 y figura 4) a procesos externos tales como deslizamientos, erosión de márgenes, y la confluencia con el colector principal de canales laterales con aporte de detritos, que abastecen el canal principal con troncos grandes y estables capaces de formar una gran acumulación transversal atrapando pedazos de madera más pequeños (Abbe y Montgomery 2003).

\section{CONCLUSIONES}

Se determinó que los residuos leñosos de gran tamaño (LWD) en el área del cauce de una cuenca que drena bosque nativo maduro en Los Andes meridionales de Chile son muy abundantes. De hecho, la cantidad de LWD en el torrente Tres Arroyos (700 y $1.500 \mathrm{~m}^{3}$ por hectárea de lecho del canal, considerando LWD en relación al volumen del cauce lleno y al área entera del canal, respectivamente) es comparable sólo con valores registrados en los bosques nativos maduros del noroeste de Norteamérica, cercanos a la costa del Océano Pacífico. La mortalidad natural de los árboles, más que los deslizamientos o la erosión de las orillas, parece ser la causa más importante de ingreso de LWD al cauce del torrente Tres Arroyos; sin embargo, los puntos donde confluyen al colector principal canales laterales de debris flow representan puntos críticos de concentración de LWD que afectan fuertemente la morfología del canal. Estas grandes cantidades de residuos leñosos ejercen una considerable influencia en la funcionalidad del flujo hídrico, formando grandes acumulaciones o tacos de valle y altos diques de troncos que imponen al canal un perfil de escalones a nivel de macroescala. Esto contribuye a la disipación de más de un cuarto de la energía potencial de la corriente de agua y al depósito de un volumen de sedimentos por lo menos del mismo orden de magnitud que la producción anual de sedimentos de la cuenca.

\section{AGRADECIMIENTOS}

Esta investigación fue financiada por el proyecto INCO-CT-2004-510735 "Epic Force" del EU (Evidence-based policy for integrated control of forested river catchments in extreme rainfall and snowmelt; Política basada en evidencias para el control integrado de las cuencas fluviales forestadas en presencia de eventos extremos). Los autores agradecen al profesor Mario Aristide Lenzi (Universidad de Padua, Italia) por el apoyo brindado a toda la investigación; a Hardin Palacios y a Paula Uyttendaele (Universidad Austral de Chile) por su ayuda logística, aporte de material informativo y apoyo en el trabajo de campo. Además se agradece al personal de CONAF de Malalcahuello por la colaboración prestada y a la Dra. Mónica Montoya por la revisión del manuscrito.

\section{REFERENCIAS}

Abbe TB, DR Montgomery. 2003. Patterns and processes of wood debris accumulation in the Queets river basin, Washington. Geomorphology 51: 81-107.

Baillie BR, TR Davies. 2002. Influence of large woody debris on channel morphology in native forest and pine plantation streams in the Nelson region, New Zealand. New Zealand Journal of Marine and Freshwater Research 36: 763-774.

Bilby RE. 1979. The function and distribution of organic debris dams in forest stream ecosystems. PhD dissertation. Division of Biological Sciences, Cornell University, USA.

Comiti F, A Andreoli, MA Lenzi, L Mao. 2006. Spatial density and characteristics of woody debris in five mountain rivers of the Dolomites (Italian Alps). Geomorphology 78 (1-2): 44-63.

CONAF-CONAMA. 1999. Catastro y evaluación de recursos vegetacionales nativos de Chile, Informe Nacional con Variables Ambientales. Proyecto CONAF-CONAMA-BIRF, Santiago, Chile, 88 p. 
Curran JH, EE Wohl. 2003. Large woody debris and flow resistance in step-pool channels, Cascade Range, Washington. Geomorphology 51: 141-157.

Darby SE, CR Thorne. 1995. Case studies and reviews: fluvial maintenance operations in managed alluvial rivers. Aquatic Conservation: Marine and Freshwater Ecosystems 5: 37-54.

DGA (Dirección General de Aguas). 2000. Cuenca experimental representativa, Reserva Forestal Malalcahuello, Cuenca Río Imperial, IX Región de la Araucanía. Informe Final S.I.T. Nº 68, Convenio Dirección General de Aguas-Universidad Austral de Chile, Facultad de Ciencias Forestales, Valdivia. Volumen 1, 116 p; Volumen 2, 196 p.

Donoso, C. 1993. Bosques templados de Chile y Argentina. Variación, estructura y dinámica. Editorial Universitaria, Santiago, Chile, 484 p.

Dudley SJ, JC Fischenich, SR Abt. 1998. Effect of woody debris entrapment on flow resistance. Journal of the American Water Resources Association 34(5): 1189-1197.

Emparan C, M Suárez, J Muñoz. 1992. Curacautín, Carta Geológica de Chile. SERNAGEOMIN, Chile.

Faustini JM, JA Jones. 2003. Influence of large woody debris on channel morphology and dynamics in steep, boulder-rich mountain streams, western Cascades, Oregon. Geomorphology 51: $187-205$.

Fuenzalida H. 1965. Geografía económica de Chile. Texto refundido. Santiago, Chile. CORFO, p. 130-267.

Gomi T, RC Sidle, RD Woodsmith, MD Bryant. 2003. Characteristics of channel steps and reach morphology in headwater streams, southeast Alaska. Geomorphology 51: 225-242.

Gurnell AM. 2003. Wood storage and mobility. In The ecology and management of wood in world rivers, Gregory SV, KL Boyer, AM Gurnell eds. American Fisheries Society, Bethesda, Maryland, p. 75-91.

Gurnell AM, H Piegay, SV Gregory, FJ Swanson. 2002. Large wood and fluvial processes. Freshwater Biology 47: 601-619.

Hechenleitner P, M Gardner, P Thomas, C Echeverría, B Escobar, P Brownless, C Martínez. 2006. Plantas ornamentales del centro-sur de Chile. Distribución, conservación y propagación. Primera edición, Trama Impresores S.A., Valdivia, Chile. 188 p.

Heede BH. 1972. Flow and channel characteristics of two high mountain streams. Research Paper RM-96, Rocky Mountain Forest and Range Experiment Station, U.S. Forest Service, Fort Collins, Colorado.

Hilderbrand RH, AD Cemly, CA Dolloff, KL Harpster. 1997. Effects of large woody debris placement on stream channel and benthic macroinvertebrate. Canadian Journal of Fisheries and Aquatic Science 54: 931-939.

Iroumé A. 1997. Estudio de los procesos hidrológicos en una cuenca experimental andina de la IX Región, Chile. Bosque 18(1): 73-81.

Iroumé A. 2003. Transporte de sedimentos en una cuenca de montaña en la Cordillera de los Andes de la Novena Región de Chile. Bosque 24(1): 125-136.

Jackson CR, CA Sturm. 2002. Woody debris and channel morphology in first- and second-order forested channels in Washington's Coast Ranges. Water Resources Research 38(9): 1177.

Jacobson PJ, KM Jacobson, PL Angermeier, DS Cherry. 1999. Transport, retention and ecological significance of woody debris within a large ephemeral river. Journal of the North American Benthological Society 18(4): 429-444.

Keim RF, AE Skaugset, DS Bateman. 2000. Dynamics of coarse woody debris placed in three Oregon streams. Forest Science 46: 13-22.

Keller EA, FJ Swanson. 1979. Effects of large organic material on channel form and fluvial processes. Earth Surface Processes and Landforms 4: 361-380.

Keller EA, T Tally. 1979. Effects of large organic debris on channel form and fluvial processes in the coastal Redwood environment. In Adjustments of the Fluvial System, Rhodes DD, GP Williams eds. Kendal-Hunt, Dubuque, IA: 169-197.

Kochel RC, DF Ritter, J Miller. 1987. Role of tree dams in the construction of pseudo-terraces and variable geomorphic response to floods in Little River Valley, Virginia. Geology 15: 718-721.

Kraft CE, DA Warren. 2003. Development of spatial pattern in large woody debris and debris dams in streams. Geomorphology 51: 127-139.

Lacey RWJ, RG Millar. 2004. Reach scale hydraulic assessment of instream salmonid habitat restoration. Journal of the American Water Resources Association 40(6): 1631-1644.

Lenzi MA, A Iroume, L Mao, H Palacios. 2004. Analysis of sediment transport in experimental Alpine and Andean catchments. In Proceedings of the $10^{\circ}$ International Symposium Interpraevent. Riva del Garda, Italy; vol 2: 77-88.

Marston RA. 1982. The geomorphic significance of log steps in forest streams. Annals of the Association of American Geographers 72(1): 99-108.

Martin DJ, LE Benda. 2001. Patterns of instream wood recruitment and transport at the watershed scale. Transaction of the American Fisheries Society 130: 940-958.

Mehagan WF. 1982. Channel sediment storage behind obstructions in forested drainage basins draining the granite bedrock of the Idaho batholith. In Sediment Budgets and Routing in Forested Drainage Basins, Swanson FJ, RJ Janda, T Dunne, DN Swanston eds. USDA Forest Service General Technical Report, PNW-141, p. 114-121.

Montgomery DR, JM Buffington. 1997. Channel-reach morphology in mountain drainage basins. Geological Society of America Bulletin 109: 596-611.

Montgomery DR, H Piégay. 2003. Wood in rivers: interactions with channel morphology and processes. Geomorphology 51: $1-5$.

Montgomery DR, BD Collins, KM Buffington, TB Abbe. 2003. Geomorphic effects of wood in rivers. In The ecology and management of wood in world rivers, Gregory SV, KL Boyer, AM Gurnell eds. American Fisheries Society, Bethesda, Maryland, p. 21-47.

Otero L. 2006. La huella del fuego. Historia de los bosques nativos y cambios en el paisaje del sur de Chile. Pehuén Editores, Santiago, Chile, 171 p.

Shields FD, CJ Gippel. 1995. Prediction of effects of woody debris removal on flow resistance. Journal of Hydraulic Engineering 121: 341-354.

Shields FD, N Morin, CM Cooper. 2004. Large woody debris structures for sand-bed channels. Journal of Hydraulic Engineering 130(3): 208-217.

Swanson FJ, RC Fredriksen. 1982. Sediment routing and budget implications for judging impacts of forestry practices. 
In Sediment Budgets and Routing in Forested Drainage Basins, Swanson FJ, RJ Janda, T Dunne, DN Swanston eds. USDA Forest Service General Technical Report, PNW-141, p. 129-137.

Swanson FJ, GW Lienkaemper, JR Sedell. 1976. History, physical effects, and management implications of large organic debris in western Oregon streams. U.S. Forest Service General Technical Report, PNW-56.

Uyttendaele GP. 2006. Procesos de transporte de sedimentos en áreas de montaña, comparación entre la cuenca del Río Cordón (Alpes, Italia) y el Torrente los Tres Arroyos (Andes, Chile). Tesis Doctorado en Ciencias Forestales, Valdivia, Chile. Facultad de Ciencias Forestales, Universidad Austral de Chile. 236 p.
Webb AA, WD Erskine. 2003. Distribution, recruitment, and geomorphic significance of large woody debris in an alluvial forest stream: Tonghi Creek, southeastern Australia. Geomorphology 51: 109-126.

Wohl E, S Madsen, L MacDonald. 1997. Characteristics of log and clast bed-steps in step-pool streams of northwestern Montana, USA. Geomorphology 20: 1-10.

Wing MG, RF Keim, AE Skaugset. 1999. Applying geostatistics to quantify distributions of large woody debris in streams. Computers and Geoscience 25: 801-807.

Zelt RB, EE Wohl. 2004. Channel and woody debris characteristics in adjacent burned and unburned watersheds a decade after wildfire, Park County, Wyoming. Geomorphology 57(3): 217-233

Recibido: 01.08 .06

Aceptado: 19.03.07 